Lingua Litteria Journal

\title{
La Transformation Des Personnages et Des Fonds Dans Le Roman "La Gloire de Mon Père" de Marcel Pagnol au film: Une Étude d'Écranisation de Pamusuk Eneste
}

\author{
Ristiani Alfiana Lestari $\bowtie$ Sunahrowi ${ }^{\bowtie}$ Ahmad Yulianto
}

Département de la Langue et la Littérature Étrangère, Faculté des Langues et des Arts, Universitas Negeri Semarang, Indonesia

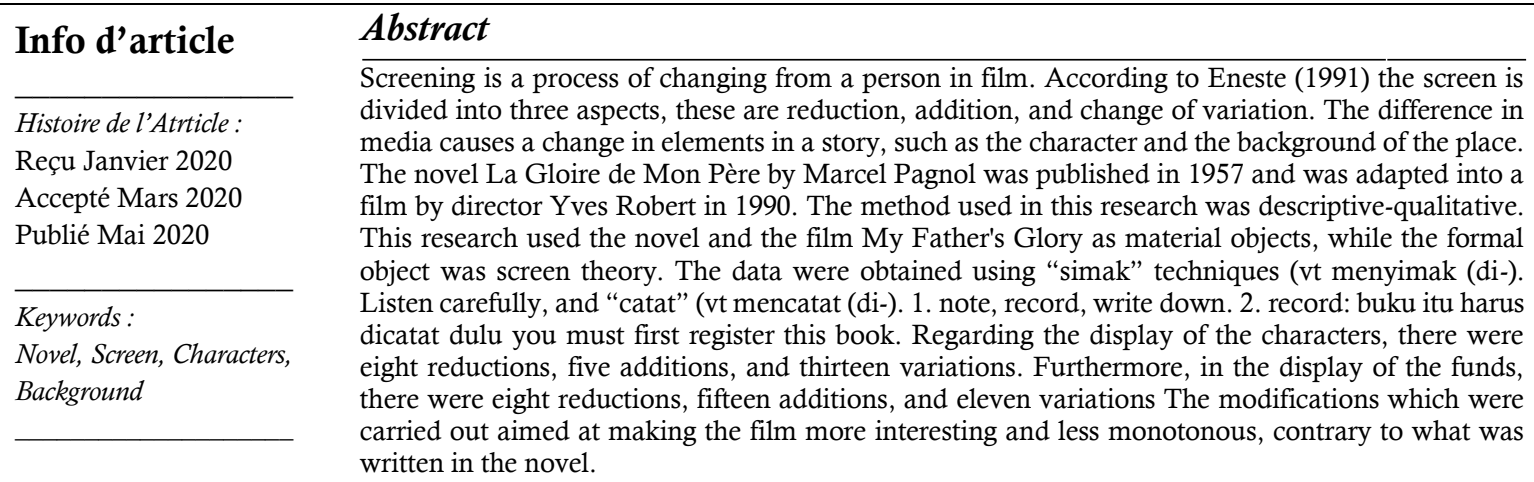

\section{Extrait}

L'écranisation est un processus de changement d'unroman en film. Selon Eneste (1991) l'écranisation est divisée en trois aspects, ces sont la réduction, l'addition, et le changement de variation. La différence de médias provoquent un changement des éléments dans une histoire, tels que le personnage et le fond de lieu. Le roman La Gloire de Mon Père de Marcel Pagnol a été publié en 1957 et a été adapté en film par le réalisateur Yves Robert en 1990. La méthode utilisée dans cette recherche était descriptive-qualitative. Cette recherche a utilisé le roman et le film La Gloire de Mon Père comme les objets matériels, alors que l'objet formel était la théorie d'écranisation. Les données ont été obtenues à partir des techniques "simak" (v.t. menyimak (di-). écouter attentivement, et "catat" (v.t. mencatat (di-). 1. noter, relever, inscrire. 2. enregistrer : buku itu harus dicatat dulu il faut d'abord enregistrer ce livre. Concernant l'écranisation des personnages, il y a eu huit réductions, cinq additions, et treize variations. Par ailleurs, dans l'écranisation des fonds, il y a eu huit réductions, quinze additions, et onze variations. Les modifications qui ont été menées visaient à rendre le film plus intéressant et moins monotone, contrairement à ce qui était écrit dans le roman.

(C) 2020 Universitas Negeri Semarang

Addresse:

ISSN 2252-6730

Gedung B4 FBS Universitas Negeri Semarang

Kampus Sekaran, Gunung Pati, Semarang, 50229 


\section{INTRODUCTION}

Selon Sumardjo et Saini (1988:3) la littérature est une expression de chaque être humain qui vient sous la forme de l'expérience, de la contemplation, du sentiment, de l'idée, et de la croyance à l'intérieur d'une description concrète qui engendre le charme de l'instrument de langage. Cela a une sens que, à travers la littérature, un auteur pourrait exprimer son point de vue vers la vie dans son environnement.

Sapardi (1979:1) a déclaré que la littérature est une association sociale qui utilise une langue comme moyen de communication. Le langage lui-même fait partie de la création sociale. La littérature montre la représentation de la vie où la vie elle-même est une réalite de l'événement social. Ainsi, celle peut être simplifié qu'une oeuvre littéraire est le resultat d'un métier de l'écrivain qui décrit l'imitation ou la représentation de ce qui se passe dans la vie humaine.

Donc, un travail de littérature peut être avantageux pour la vie, car une oeuvre littéraire peut donner une certaine réalisation aux lecteurs sur la vérité de la vie, même si elle est écrite dans un récit fictif.

La chercheuse se concentre sur un roman intitulé La Gloire de Mon Père de Marcel Pagnol qui était le premier parti de la série Souvenirs d'Enfance, ce travail a été adapté dans un film réalisé par Yves Robert. "La Gloire de Mon Père" raconte la vie de Marcel Pagnol qui est né à Aubagne mais être élévé à Marseille. Son père, Joseph, était enseignant dans une école publique athée d'Aubagne alors que sa mère, Augustine, était autrefois tailleur. En été 1904, sa famille décida de vivre dans une villa nommée Bastide Neuve, au sommet d'une colline. Un jour, Joseph et Jules (l'oncle de Pagnol) projetaient d'aller chasser dans la forêt mais sans les enfants parce que cela pourrait les mettre en péril. Marcel, qui avait entendu parler de ce projet, proposa de force aux vieillards qu'il s'engageait et ne pas le dire à Paul, son petit frère. Le jour de la chasse, Marcel réalisait qu'il était exclu de l'aventure et se sentait découragé, il a décide de suivre secrètement. Plus tard, il a été ravi d'assister à un spectacle où son père pourrait abattre une paire d'oiseaux alors quand en fait il était une sorte d'homme qui ne casserait jamais une plume. Dans leur retour triomphal, la réussite de son père devient le sujet brûlant du quartier, cela a transformé la déception de Marcel en gloire.

Le film "La Gloire de Mon Père" d'Yves Robert est devenu le deuxième sujet de cette recherche. Le chercheur a choisi ce film car c'était la seule adaptation qui était basée sur le roman qui allait avec le même titre. Ce film a été publié en 1990 en France avec la durée de 110 minutes.

\section{MÉTHODE DE RECHERCHE}

Le type d'approche utilisé dans cette recherche est l'approche objective. L'approche objective est une approche où elle se concentre entièrement sur la littérature. Une approche qui est perçue à travers son existence est construite sur la convention de littérature en cours (Fananie, 2002:112). L'approche objective centre toute la concentration uniquement sur les éléments, appelés l'analyse intrinsèque (Ratna, 2010:73).

La méthode appliquée dans cette recherche est descriptive qualitative. Moleong (2002:3) a défini que la recherche qualitative fonctionne comme la recherche procédurale qui produit des données descriptives sous la forme de phrases écrites ou orales provenant des personnes et des sujets qui peuvent être surveillé.

Ratna (2010:53) a expliqué que l'analyse des données d'une manière descriptive pourrait être menée en décrivant les faits qui ont ensuite adhéré à l'analyse. Les données obtenues par l'enregistrement sont ensuite identifiées et classées dans la catégorie qui a été déterminée. Plus tard, les données mentionnées précédemment seront interprétées en les connectant avec le texte où les données sont occupées. En outre, dans une autre inférence, est de résumer les données qui ont été cataloguées à faire dans ses descriptions en conformité avec l'étude de recherche.

Selon la description au-dessus, l'objectif de cette recherche est de déterminer la réduction, l'addition, et le changement de variation qui apparaissent dans le fond de lieu et la caractérisation dans la version cinématographique de "La Gloire de Mon Père".

\section{RÉSULTAT ET DISCUSSION}

Selon Nurgiyantoro (2005:165), le terme caractère désigne les personnes qui font l'acte. La nature, la caractérisation, et le caractère soulignent la caractéristique et le comportement des 
personnages interprétés par les lecteurs. C'est plus pour montrer la qualité personelle d'un personnage. Les caractères fictifs peuvent être différenciés en plusieurs noms basés sur l'angle dans lequel ils ont été créés. Deux d'entre eux sont le caractère principal et le caractère secondaire.

Le caractère principal est un personnage dont les histoires sont les plus éminentes dans un scénarios. Le caractère principal est le personnage qui a été écrit beaucoup. Car le caractère principal est une personne qui apparaît beaucoup et qui connecté toujours avec d'autres caractères, ils déterminent entièrement le développement de l'intrigue. En outre, à coté de l'apparence du caractère principal est le caractère secondaire. L'apparence de caractère secondaire dans tout le scénario doit être moindre, meme sa présence n'est pas prioritaire s'il y a une connexion avec le caractère principal directement ou non (Nurgiyantoro 2013:259).

Basés sur des charactères présents dans le roman et dans le film, on pourrait comprendre que, dans le processus d'adaptation, on pourrait aussi bien les modifier. Dans la visualisation du film, il y a des charactères qui sont réduits ou coupés. En plus de la réduction, il y a un autre processus appelé addition où des caractères qui ne sont pas présents dans le roman peuvent être présentés dans la version cinématographique. Avec les deux aspects mentionées au-dessus, il y a aussi le changement de variation sur les charactères dans le film, à partir de leurs descriptions, ou de leurs extérieurs, ainsi que leurs attitudes.

Le premier aspect à discuter est la réduction. Il y a 8 réductions dans cette recherche et l'une d'entre elles est tante Marie. Tante Marie est la soeur aînée de Joseph, dans le roman elle a été écrite comme quelqu'un qui a aidé à calmer la jeune Augustine qui est panique au sujet de sa grossesse. Tante Marie emmena Augustine chez elle à la plage afin de rendre son âme en paix. Cet plan a réussi jusquà ce que la douleur a frappé l'abdomen d'Augustine, elle a demandé de l'aide et ordonne à Marie de la ramener à la maison.

Marie l'a ramenée à Aubagne, et c'est la fin de son histoire. Elle n'apparaissait plus dans le reste du roman et elle n'est pas sortie dans la version cinématographique. Marie a été simplement coupée du film en raison de son insignificance pour le développement de l'intrigue, donc elle a été éliminée.

Le deuxieme est l'addition. Il y a 5 additions trouvées et l'un d'eux est Lili de Belons, il a donné une contribution significative au développement de l'intrigue. Lili ne serait pas trouvé dans le roman comme il est un nouveau charactère qui est apparu seulement dans le film.

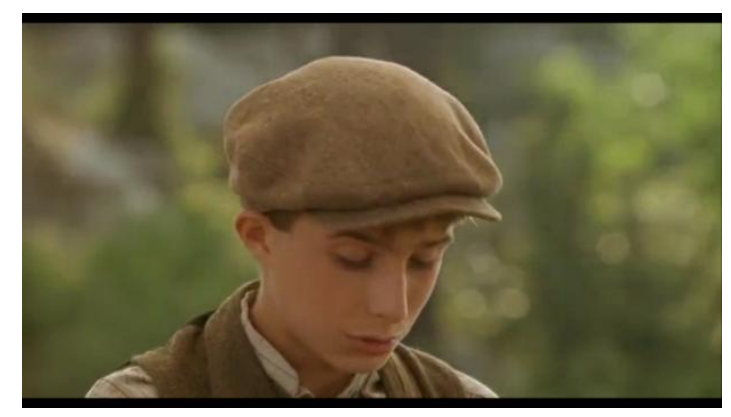

C'est une photo de Lili. Il est apparu à la mi-temps du film. Lili rencontra Marcel pour la première fois lorsque Marcel se perdit au milieu d'un vaste désert. Marcel essayait de retracer le pas que son père et son oncle avaient laissé derrière lui, mais il n'a rien trouvé, il a continué à marcher et s'est retrouvé dans un désert. Marcel a trouvé un piège avec un oiseau à l'intérieur, il a essayé d'approcher pour voir quand soudainement un garçon a crié que l'oiseau était le sien. Ils sont devenus amis plus tard. Marcel était tellement excité quand Lili lui proposa son idée de devenir un ermite qui vivait dans une caverne. Marcel l'accepta, mais il avait peur de se séparer de sa famille. Lili a compris et leur plan a été annulé. C'était le dernier jour de vacances de Marcel et il a dû retourner à la ville. Lili salua Marcel et ils étaient tristes.

Le dernier aspect est le changement de variation. Cet aspect a établi 13 changements, le plus parmi les deux autres. Les changements dans chaque caractère inclus leur éxterieur, comme la différence entre leurs vêtements portés dans le roman et le film, et leur attitude. Marcel, comme le caractère principal, a eu beaucoup de changements.

L'hypothèse de Mangiapan me parut démontrée, et j'embrassai ma mère tendrement en songeant à ses souffrances au moment où il avait fallu déboutonner son nombril. (Pagnol 1957: 15) 


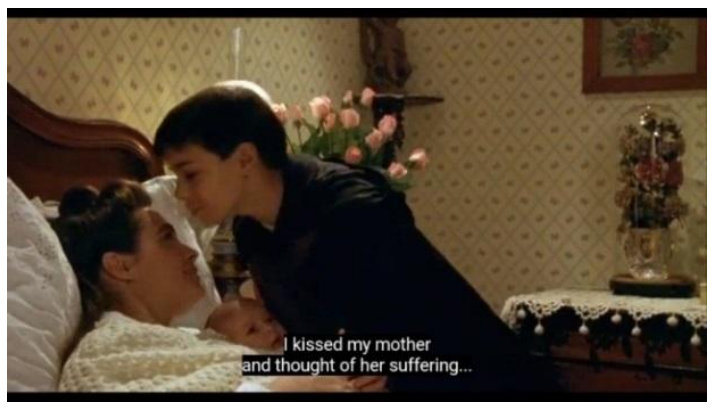

La photo ci-dessus montre la différence d'attitude de Marcel. C'était une scène où lui mère, Augustine, a donné naissance à sa plus jeune soeur. Après la naissance, elle était encore faible et elle resta sur le lit en tenant le bébé dans ses bras. Marcel, témoin de cette vue, entra dans la pièce et se dirigea vers elle. Il lui donna un bisou sur le front, c'est différent de ce qui était écrit dans le roman. À partir de la citation, il a été dit que Marcel est venu serrer tendrement sa mère en pesant aux difficultés qu'elle avait quand elle avait accouché.

Le lendemain matin, dès huit heures, nous étions prêts, et déjà revêtus du costume des vacances: culottes de toile écrue, et chemises à manches courtes, blanches, mais ornées de cravates bleues. (Pagnol 1957: 24)

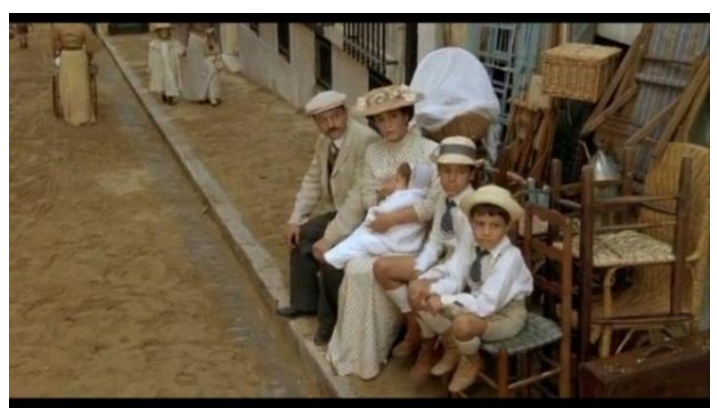

Le changement de variation peut être vu de l'image au-dessus. La photo montre que la famille s'assied sur la façade de leur maison en attendant le paysan et sa charrette viennent les chercher. On peut voir que Marcel et Paul portaient une tenue identique, une chemise blanche à manches longues, une cravate grisâtre, un chapeau tissé, une paire de chaussures et des chaussettes qui atteignent leurs mollets. Les manches sur leur chemises doivent être courtes mais dans le film elles sont longues. Une autre changement a été les chaussettes et des chaussures, elle n'était pas particulièrement expliquées en le roman. Cela signifie que le processus de changement de Marcel inclut la variation de son éxterieur.

Selon Abrams cité de Nurgiyantoro (2013:314) le fond est divisé en trois aspects principaux. Ces trois aspects imprègnent dans le fond de lieu, le fond du temps, et le fond en relation avec la vie sociale où les histoires écrites se déroulent. Pour réduir la discussion, cette recherche se concentrera sur le fond de lieu.

Ainsi que ce qui a été fait dans l'aspect de la caractérisation, ici sera effectué le changement du fond de lieu. Dans la visualisation du film, certains fonds de lieu ont été réduits, ajoutés, ou même modifiés.

Le premier aspect est la réduction. Il y avait 7 réductions trouvées dans le film et l'un d'eux était le bord de mer où se trouve la maison de tante Marie. Augustine était écrit marchant le long de plage pour profiter de la brise de la mer, se dorer au soleil de janvier, tout en regardant au loin dans la mer où naviguaient les bateaux des pêcheurs. Elle alluma en feu de joie puis s'assit l'avant, de tricoter des vêtements pour le nouveau-né, tante Marie plissa des couches en sérénade d'une voix claire. Malheureusement, cet extrait n'a été pas inclus dans le film.

Passer à l'aspect suivant est l'addition. 15 ajouts de places ont été faits dans le film et la maison de Marcel à Saint-Loup a appartenu à la liste. Saint-Loup était la deuxième destination de l'histoire de la vie de Marcel. Dans le roman a été écrit que le jeune Marcel était capable de lire plus tôt que prévu, chaque fois qu'Augustine devait faire des courses, elle le faisait asseoir dans la classe de Joseph, donc l'endroit présenté était seulement la classe où cette histoire se passait at le reste de la maison n'a 
été pas spécifiquement écrit. Au contraire, il y a eu une altération dans la version cinématographique où la façade, la salle à manger, et la chambre à coucher sont apparues.

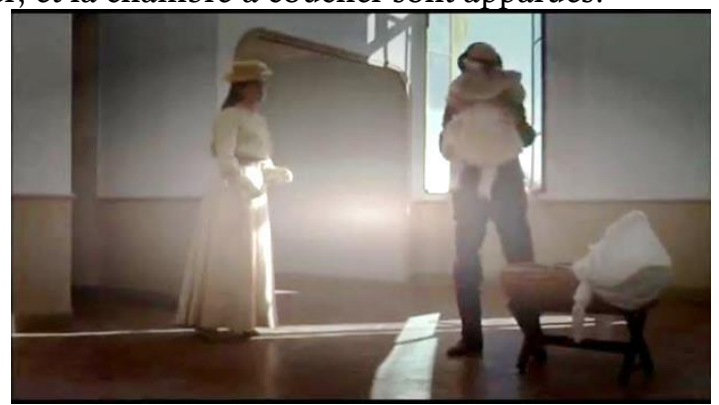

Vu de la photo au-dessus, Joseph souleva le petit Paul de son berceau en bois et Augustine se tenait à côté de lui prêt à prendre le bébé dans ses bras. La salle était déjà vacante car c'était le jour de leur déménagement à Marseille.

Il y avait un passsage où Marcel et sa famille attendaient que le paysan vienne ramasser les marchandises qui seraient apportées à la villa. Ils étaient impatients, attendaient nerveusement devant la fenêtre. C'est un changement de variation.

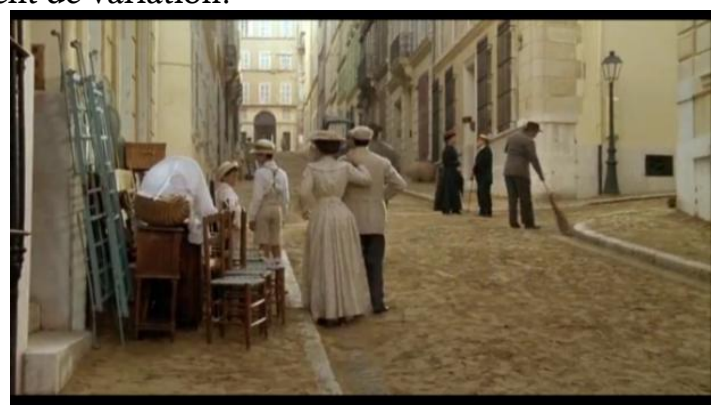

La photo au-dessus montre que la famille de Marcel attendait au bord de la rue. Cette changement était techniquement acceptable car la situation de leur maison nétait pas similaire à celle du roman, leur maison en film était un appartement au lieu d'un seul bâtiment, donc il serait irréalisable s'ils attendaient à l'intérieur de la maison.

\section{CONCLUSION}

En général, un film reçoit des opinions différents, de ceux qui aiment et ceux qui n'en aiment pas. Le film adapté d'un travail littéraire les recevra également, c'est inévitable car plusieurs résultats sont décevants.

La différence fondamentale entre lire un roman et regarder un film réside dans le travail d'imagination. Quand les gens lisent un roman, ils sont forcé d'explorer plus de leur imagination qui est infinie. C'est different quand les gens regardent un film où la visualisation des acteurs, des fonds, et même de scenes sont montrés clairement, donc les observateurs n'ont pas à deviner comment chaque scène se présentera, livrera-t-elle ou non.

La thème du film La Gloire de Mon Père réalisé par Yves Robert est basé sur ce qui est écrit en roman, mais il y a quand même quelques changements. Dans l'aspect de characterisation a été trouvé 8 réductions, 5 additions, et 13 changements de variation. En l'aspect du fond, il y avait 7 réductions, 15 additions, et 11 changements de variation.

Un travail d'adaptation (le film) ne sera jamais semblable à sa source (le roman) car ces deux pieces nées de deux créateurs différents, d'où les idées dans les oeuvres sont différentes aussi. Dans ce cas, les ajustements sont montrées à partir des changements dans la caractérisation et le fond qui est basé sur l'altération de l'intrigue entre le roman et le film. Néanmoins, la theme de La Gloire de Mon Père n'est pas déformé donc on peut dire que tous les changements se produisent dans le film sont acceptables.

\section{BIBLIOGRAPHIE}

Damono, Sapardi Djoko. 1978. Sosiologi Sastra (Sebuah Pengantar Ringkas).Jakarta: Depdikbud. 
Eneste, Pamusuk. 1991. Novel dan Film. Flores: Penerbit Nusa Indah.

Fananie, Zainuddin. 2002. Telaah Sastra. Surakarta: Muhammadiyah University Press.

Moleong, Lexy J. 2010. Metode Penelitian Kualitatif. Bandung: Remaja Rosdakarya.

Nurgiyantoro, Burhan. 2005. Teori Pengkajian Fiksi. Yogyakarta: Gadjah Mada University Press.

Nurgiyantoro, Burhan. 2013. Teori Pengkajian Fiksi (Edisi Revisi). Yogyakarta: Gadjah Mada University Press. Pagnol, Marcel. 1957. La Gloire de Mon Père. Paris: Pastorelly.

Ratna, Nyoman Kutha. 2010. Teori, Metode, Dan Tekhnik Penelitian Sastra. Yogyakarta: Pustaka Pelajar.

Sumardjo, Jacob \& Saini K.M. 1988. Apresiasi Kesusastraan. Jakarta: Gramedia.

Wellek, Rene \& Austin Warren. 1956. Theory of Literature. Edisi ke-3. New York: A Harvest Book. 